\title{
Uso de modelos epidemiológicos para estimar la incidencia de caries dental y enfermedad periodontal en embarazadas chilenas
}

\author{
Use of epidemiological models to estimate the incidence \\ of dental caries and periodontal disease in Chilean pregnant women
}

\author{
Corsini Muñoz $\mathrm{G}^{1}$, Zaror Sánchez $\mathrm{C}^{2}$, Vallejos Vallejos $\mathrm{C}^{3}$
}

\section{RESUMEN}

Objetivo: Determinar la incidencia de caries y enfermedad periodontal en embarazadas chilenas mediante un modelo de incidencia, prevalencia y mortalidad (IPM). Material y Método: Se construyó un modelo IPM con la prevalencia estimada en la Encuesta Nacional de Salud del año 2003 y los datos del Estudio de Carga de Enfermedad en Chile en el año 2007. Para efectos de estimar la población de mujeres embarazadas, se utilizó como variable de aproximación, los partos por edad de las mujeres según la información de los nacimientos registrado por el Instituto de Nacional de Estadística (INE). Los datos fueron ingresados en el programa DisMod para determinar las incidencias y comprobar la consistencia interna del modelo. Resultados: La incidencia de caries estimada por el modelo fue de 86.870 nuevos casos y de 7.983 casos incidentes al año para la enfermedad periodontal, en un universo estimado de 230.831 mujeres embarazadas. Conclusión: Los modelos epidemiológicos son una herramienta útil para determinar la epidemiología de cualquier enfermedad, en donde la estimación de la incidencia constituye un problema y estudios de investigación en campo serían muy costoso, de larga duración y/o en donde la problemática ética del estudio sería inaceptable.

Rev. Clin. Periodoncia Implantol. Rehabil. Oral Vol. 5(2); 74-77, 2012.

Palabras clave: Incidencia, epidemiologia, modelo, embarazo, caries, enfermedad periodontal.

\section{ABSTRACT}

Objectives: To determine the incidence of caries and periodontal disease in pregnant Chilean women through an incidence, prevalence and mortality model (IPM). Material and Methods: An IPM model was constructed with the prevalence estimated the National Health Survey 2003 and data from the Burden of Disease study in Chile in 2007. The childbirths by age of women, according to the information from the National Statistics Institute, were used to estimate the population of pregnant women. Data were entered into the DisMod program to determine the incidence and check the internal consistency of the model. Results: The estimated incidence of caries by the model was of 86.870 new cases and 7.983 incident cases per year for periodontal disease, in a total of 230.831 pregnant women. Conclusion: Epidemiological models are a useful tool to determine the epidemiology of any disease, where the estimation of the incidence is problematic and field research would be very expensive, long-term and where the ethic problems of the study would be unacceptable.

Rev. Clin. Periodoncia Implantol. Rehabil. Oral Vol. 5(2); 74-77, 2012.

Key words: Incidence, epidemiology, model, pregnancy, caries, periodontal disease.

\section{INTRODUCCIÓN}

Durante el embarazo ocurre una serie de alteraciones fisiológicas y conductuales que inducen a cambios en el entorno oral, lo cual predispone a un incremento en la incidencia de diferentes patologías de la cavidad oral, entre otros, caries dental, gingivitis, tumores orales, etc. ${ }^{(1)}$.

Hay bastante evidencia científica que sustenta la relación entre embarazo y condición periodontal(2-4). Si bien el embarazo no causa enfermedad periodontal, las fluctuaciones en los niveles de estrógeno y progesterona pueden exacerbar una enfermedad periodontal prexistente $^{(5,6)}$.

Hay consenso en la literatura que las pacientes embarazadas tienen un deterioro de su salud periodontal mayor en relación a las mujeres no embarazadas, en especial de la gingivitis, con prevalencias que fluctúan entre el $30 \%$ al $100 \%(7)$.

Aunque hay escasa literatura que relaciona el embarazo con caries dental, es sabido que alteraciones conductuales, fisiológicas, cambios dietarios (aumento en el consumo de carbohidratos), el aumento de ácidos en la boca asociado a vómitos y acidez de la saliva, junto con la disminución del flujo salival, predispondrían a un aumento en la incidencia de caries. Sin embargo, la evidencia no es concluyente al respecto con resultados contradictorios ${ }^{(8,9)}$. La prevalencia de caries en embarazadas se estima cercana al $100 \% \%^{(9-11)}$

Describir cuantitativamente la epidemiología de cualquier enfermedad crónica, como la caries y la enfermedad periodontal es frecuentemente difícil de realizar, requiere largo tiempo y casi siempre a un alto costo(12).

En respuesta a esto, la epidemiología ha desarrollado sofisticados métodos para medir las causas y los patrones de enfermedad en las poblaciones. Sin embargo, para muchas enfermedades en innumerables países, la información sobre incidencia, prevalencia y mortalidad sobre alguna enfermedad específica son frecuentemente incompletas, no confiables o inexistentes. En este caso, es posible complementar los datos derivados de las relaciones causales entre las distintas variables que describen el proceso de enfermedad, mediante un modelo teórico de incidencia, prevalencia, mortalidad y remisión ${ }^{(13)}$.

El modelo se basa en un conjunto de ecuaciones diferenciales

1. Magíster en Epidemiología Clínica. Especialista en Endodoncia. Docente del Departamento de Odontología Integral Adulto. Docente de la Escuela de Odontología de la Facultad de Odontología. Docente del Programa de Magíster en Epidemiología Clínica del Centro de Excelencia CIGES. Universidad de La Frontera. Temuco, Chile.

2. Magíster en Epidemiología Clínica. Especialista en Odontopediatría. Docente del Programa de Especialización en Odontopediatría, Facultad de Odontología. Universidad de La Frontera. Temuco, Chile.

3. Magíster en Epidemiología Clínica. Médico Cirujano, Especialista en Ginecología y Obstetricia. Director Magister en Epidemiología Clínica. Centro de Excelencia CIGES, Facultad de Medicina. Universidad de La Frontera. Temuco, Chile.

Correspondencia autor: Gilda Corsini Muñoz. gcorsini@ufro.cl. Dpto. Odontología Integral Adulto, Facultad de Odontología. Universidad de La Frontera. Temuco, Chile. Trabajo recibido el 28/12/2011. Aprobado para su publicación el 12/07/2012. 
que describen el proceso de la enfermedad, requiriendo como insumo un mínimo de 3 variables de entrada. El conjunto de ecuaciones se inserta en un software llamado DisMod ${ }^{(13)}$.

Este software se ha utilizado ampliamente para complementar los datos que faltan en las bases de datos y la coherencia en la fuerza en los datos que están disponibles. Con información como la prevalencia, remisión y mortalidad, DisMod es capaz de calcular la epidemiología completa de la enfermedad, incluyendo la incidencia.

Tanto la incidencia como la prevalencia son datos de una gran importancia a la hora de planificar los recursos necesarios en un sistema sanitario, ya que nos acercan a una estimación del número potencial de usuarios que pueden acceder al sistema de salud, así como también, determinar cómo se distribuyen y evolucionan en la población las enfermedades $u$ otros fenómenos.

El objetivo de este estudio fue determinar la incidencia de caries y enfermedad periodontal en embarazadas chilenas a partir de la prevalencia y mortalidad previamente estimadas de la población chilena en general, usando el programa epidemiológico DisMod, con el propósito de obtener información útil para el posterior estudio Costo-Efectividad de Intervenciones en Salud para el subgrupo de embarazadas requerido por el Ministerio de Salud de Chile.

\section{MATERIAL Y MÉTODO}

Se realizó un modelo teórico de incidencia, prevalencia y mortalidad (IPM), sobre la historia natural de las enfermedades odontológicas con el fin de determinar la incidencia de caries dental y enfermedad periodontal en embarazadas en Chile en el año 2009.

La principal ventaja de estos modelos es que consideran que la incidencia, prevalencia y mortalidad están relacionadas a través de la cadena causal del proceso de enfermedad. Esto quiere decir que cualquier enfermedad fue antes un caso incidente o que cualquier muerte fue antes un caso prevalente.

\section{Población}

Para efectos de estimación de la población de mujeres embarazadas, se utilizó como variable de aproximación los partos por edad de las mujeres de acuerdo a la información de los nacimientos registrado por el Instituto de Nacional de Estadística al 30 de Junio del año 2009 (INE) ${ }^{(14)}$.

Asumiendo que las características dentales de las mujeres por grupos de edad son similares a las características de salud oral de la mujer embarazada, se estimó la prevalencia de caries y enfermedad periodontal con los datos de la Encuesta Nacional de Salud de Chile del $2003^{(15)}$ y del Estudio de Carga de Enfermedad del año 2007 ${ }^{(16)}$.

\section{Modelo}

Se construyó un modelo con la prevalencia estimada en la Encuesta Nacional de Salud del año 2003 y los datos del Estudio de Carga de Enfermedad del año 2007, corregidos de acuerdo al grupo de edad de mujeres en capacidad de reproducción.

Se definió caries y enfermedad periodontal de acuerdo a la Clasificación Internacional de Enfermedades en su décima versión (CIE 10), puesto que es la utilizada en la Encuesta de Salud y en el Estudio de Carga de Enfermedad.

CIE 10 cataloga a la caries dental dentro de las enfermedades de la cavidad bucal, de las glándulas salivales y de los maxilares, las que a su vez se clasifican en la categoría de enfermedades del sistema digestivo. La categoría de caries dental, codificada como K02, incluye a la caries limitada al esmalte o mancha blanca (código K02.0), caries de la dentina (código K02.1), caries del cemento (código K02.2), caries detenida (código K02.3), odontoclasia (código K02.4), otras caries dentales (código K02.8) y caries no especificadas (código K02.9) ${ }^{(17)}$.

La categoría de gingivitis y enfermedades periodontales, codificada como K05 incluye: gingivitis aguda (código K05.0), gingivitis crónica (código K05.1), periodontitis aguda (código K05.2), periodontitis crónica (código K05.3), periodontosis (código K05.4), otras enfermedades periodontales (código K02.8) y enfermedades del periodonto no especificadas (código K05.9)(17).

Para el modelo se consideró que la caries dental es una condición progresiva y que no mejora espontáneamente, por lo que se modeló con remisión cero. En el caso de la enfermedad periodontal, si bien es una condición progresiva, se consideró una remisión espontánea de un $30 \%$.

Dada las características de las patologías orales en estudio, y de acuerdo a la evidencia científica disponible se consideró que para este modelo la mortalidad de ambas condiciones es cero.

\section{Plan de Análisis}

Los datos fueron ingresados en el programa DisMod® (World Health Organization) para determinar las incidencias y comprobar la consistencia interna del modelo, de acuerdo a los parámetros descritos anteriormente.

\section{RESULTADOS}

La prevalencia de caries en el total de mujeres en Chile asciende a 389.9 por 1.000 , con un número de 3.235 .374 casos prevalentes. La incidencia de caries en el mismo grupo de mujeres fue de 335.9 casos por 1.000 mujeres al año 2009 , lo que significa un total de 1.661 .567 casos incidentes al año (Tablas 1 y 2).

En el caso de la enfermedad periodontal la prevalencia en la población general de mujeres fue de 83.6 casos por 100.000 mujeres, con un número total de 693.339 casos anuales prevalentes y la incidencia fue de 31.26 por 1.000 , lo que significa 226.980 casos incidentes por año (Tablas 3 y 4 ).

Para calcular los datos de incidencia de caries y enfermedad periodontal en mujeres embarazadas que fue el objetivo de este estudio, se utilizó la información del número de partos ocurridos en Chile según INE al 30 de junio del año 2009, en donde se estimó un universo de 230.831 mujeres embarazadas, siendo el grupo de 20 a 44 años el con mayor número de partos, con un total de 194.383 gestantes (Tabla 5).

Según lo anteriormente expuesto la incidencia de caries fue de 86.870 nuevos casos de caries en el subgrupo de embarazadas, con una duración promedio de la enfermedad de 1.9 años y una mortalidad igual a cero (Tabla 6).

En el caso de la enfermedad periodontal en mujeres embarazada, se esperan 7.983 casos incidentes al año, con una duración promedio de la enfermedad de 3.2 años y mortalidad de 0 (Tabla 7 ).

Tabla 1. Datos epidemiológicos de caries dental en mujeres. Tasas Chile 2009

\begin{tabular}{|c|c|c|c|c|}
\hline Edad & $\begin{array}{c}\text { Incidencia } \\
\text { (tasas * 1000) }\end{array}$ & $\begin{array}{c}\text { Prevalencia } \\
\text { (tasas * 1000) }\end{array}$ & $\begin{array}{c}\text { Duración } \\
\text { (años) }\end{array}$ & $\begin{array}{c}\text { Mortalidad } \\
\text { (tasas * 1000) }\end{array}$ \\
\hline 0 & 0.0 & 0.0 & 0.0 & 0 \\
\hline $1-9$ & 323.4 & 338.7 & 2.0 & 0 \\
\hline $10-19$ & 366.4 & 419.2 & 2.0 & 0 \\
\hline $20-44$ & 378.2 & 430.3 & 2.0 & 0 \\
\hline $45-49$ & 355.2 & 416.9 & 2.0 & 0 \\
\hline $50-54$ & 225.0 & 323.4 & 1.9 & 0 \\
\hline $55+$ & 141.3 & 234.7 & 1.6 & 0 \\
\hline Todos & $\mathbf{3 3 6 . 0}$ & $\mathbf{3 8 9 . 9}$ & $\mathbf{2 . 0}$ & $\mathbf{0}$ \\
\hline
\end{tabular}

Tabla 2. Datos epidemiológicos de caries dental en mujeres. Número de casos Chile 2009.

\begin{tabular}{|c|c|c|c|c|}
\hline Edad & $\begin{array}{c}\text { Incidencia } \\
\left(\mathbf{N}^{\circ} \text { absoluto) }\right.\end{array}$ & $\begin{array}{c}\text { Prevalencia } \\
\left(\mathbf{N}^{\circ} \text { absoluto) }\right.\end{array}$ & $\begin{array}{c}\text { Duración } \\
\text { (años) }\end{array}$ & $\begin{array}{c}\text { Mortalidad } \\
\text { (número) }\end{array}$ \\
\hline 0 & 3.0 & 1.0 & 2.0 & 0 \\
\hline $1-9$ & 241792.0 & 382976.0 & 2.0 & 0 \\
\hline $20-44$ & 672940.0 & 1343604.0 & 2.0 & 0 \\
\hline $45-49$ & 290035.0 & 583914.0 & 2.0 & 0 \\
\hline $50-54$ & 115646.0 & 245609.0 & 1.9 & 0 \\
\hline $55+$ & 35288.0 & 76583.0 & 1.6 & 0 \\
\hline All ages & $\mathbf{1 6 6 1 5 6 7 . 0}$ & $\mathbf{3 2 3 5 3 7 4 . 0}$ & $\mathbf{2 . 0}$ & $\mathbf{1}$ \\
\hline
\end{tabular}

* Duración promedio de la patología antes de revertir calculada por el programa. 
Tabla 3. Datos epidemiológicos de enfermedad periodontal en mujeres. Tasas Chile 2009.

\begin{tabular}{|c|c|c|c|c|}
\hline Edad & $\begin{array}{c}\text { Incidencia } \\
\text { (tasas * 1000) }\end{array}$ & $\begin{array}{c}\text { Prevalencia } \\
\text { (tasas * 1000) }\end{array}$ & $\begin{array}{c}\text { Duración } \\
\text { (años) }\end{array}$ & $\begin{array}{c}\text { Mortalidad } \\
\text { (tasas * 1000) }\end{array}$ \\
\hline 0 & 0.0 & 0.0 & 0.0 & 0 \\
\hline $1-9$ & 0.0 & 0.0 & 0.0 & 0 \\
\hline $10-19$ & 1.1 & 2.5 & 3.3 & 0 \\
\hline $20-44$ & 40.8 & 105.0 & 3.3 & 0 \\
\hline $45-49$ & 56.2 & 149.2 & 3.3 & 0 \\
\hline $50-54$ & 48.6 & 143.6 & 3.1 & 0 \\
\hline $55+$ & 45.5 & 133.7 & 2.4 & 0 \\
\hline Todos & $\mathbf{3 1 . 3}$ & $\mathbf{8 3 . 6}$ & $\mathbf{3 . 2}$ & $\mathbf{0}$ \\
\hline
\end{tabular}

Tabla 4. Datos epidemiológicos de enfermedad periodontal en mujeres. Número de casos Chile 2009.

\begin{tabular}{|c|c|c|c|c|}
\hline Edad & $\begin{array}{c}\text { Incidencia } \\
\text { (n absoluto) }\end{array}$ & $\begin{array}{c}\text { Prevalencia } \\
\text { (n absoluto) }\end{array}$ & $\begin{array}{c}\text { Duracion } \\
\text { (años) }\end{array}$ & $\begin{array}{c}\text { Mortalidad } \\
\text { (número) }\end{array}$ \\
\hline 0 & 0.0 & 0.0 & 0.0 & 0 \\
\hline $1-9$ & 0.0 & 0.0 & 0.0 & 0 \\
\hline $10-19$ & 1605.0 & 3659.0 & 3.3 & 0 \\
\hline $20-44$ & 113897.0 & 327946.0 & 3.3 & 0 \\
\hline $45-49$ & 67008.0 & 208992.0 & 3.3 & 0 \\
\hline $50-54$ & 31595.0 & 109111.0 & 3.1 & 0 \\
\hline $55+$ & 12875.0 & 43631.0 & 2.4 & 0 \\
\hline Todos & $\mathbf{2 2 6 9 8 0 . 0}$ & $\mathbf{6 9 3 3 3 9 . 0}$ & $\mathbf{3 . 2}$ & $\mathbf{0}$ \\
\hline
\end{tabular}

* Duración promedio de la patología antes de revertir calculada por el programa.

Tabla 5. Número de partos por edad según Instituto Nacional de Estadística Chile 2009.

\begin{tabular}{|c|c|}
\hline $\begin{array}{c}\text { Rango de edad de } \\
\text { mujeres }\end{array}$ & N abosoluto \\
\hline $10-19$ & 36078 \\
\hline $20-44$ & 194383 \\
\hline $45-49$ & 364 \\
\hline $50-54$ & 6 \\
\hline $55+$ & 0 \\
\hline Total & $\mathbf{2 3 0 8 3 1}$ \\
\hline
\end{tabular}

Fuente: INE, 2009.

Tabla 6. Incidencia de caries dental en Chile en mujeres embarazadas. Número de casos Chile 2009.

\begin{tabular}{|c|c|c|}
\hline Edad & Población & $\begin{array}{c}\mathbf{N}^{\circ} \text { casos } \\
\text { incidentes }\end{array}$ \\
\hline 0 & - & 0 \\
\hline $1-9$ & - & 0 \\
\hline $10-19$ & 36078 & 13217 \\
\hline $20-44$ & 194383 & 73522 \\
\hline $45-49$ & 364 & 129 \\
\hline $50-54$ & 6 & 1 \\
\hline $55+$ & - & - \\
\hline Total & $\mathbf{2 3 0 8 3 1}$ & $\mathbf{8 6 8 7 0}$ \\
\hline
\end{tabular}

Tabla 7. Incidencia de enfermedad periodontal en Chile en mujeres embarazadas. Número de casos Chile 2009.

\begin{tabular}{|c|c|c|}
\hline Edad & Población & $\begin{array}{c}\mathbf{N}^{\circ} \text { casos } \\
\text { incidentes }\end{array}$ \\
\hline 0 & - & 0 \\
\hline $1-9$ & - & 0 \\
\hline $10-19$ & 36078 & 40 \\
\hline $20-44$ & 194383 & 7922 \\
\hline $45-49$ & 364 & 20 \\
\hline $50-54$ & 6 & 0 \\
\hline $55+$ & - & - \\
\hline Total & $\mathbf{2 3 0 , 8 3 1}$ & $\mathbf{7 , 9 8 3}$ \\
\hline
\end{tabular}

\section{DISCUSIÓN}

Describir los indicadores epidemiológicos de cualquier enfermedad, como la incidencia, prevalencia y mortalidad por edad y sexo, son insumos esenciales y básicos para la realización de estudios de carga de enfermedad y análisis de costo-efectividad en ciertas intervenciones. Así como también es importante para la planificación de los recursos asistenciales a nivel nacional y regional(12).

Dado que la incidencia de caries y enfermedad periodontal en embarazadas no son conocidas en nuestro país, adquiere relevancia el uso de este tipo de modelos para estimar estos parámetros epidemiológicos, dada su importancia sanitaria, ya comentada.

Para calcular la incidencia en la gran mayoría de las enfermedades, los estudios deben tener tamaños muestrales lo suficientemente grandes y un seguimiento muy prolongado en el tiempo, lo cual los hace difíciles y costosos. A consecuencia de ello, la mayoría las observaciones publicadas son poco fiables ${ }^{(18)}$.

Esta limitación es especialmente cierta para las estimaciones de incidencia, de los cuales hay escasos o prácticamente ningún estudio que puedan ser generalizable a poblaciones específicas.

La relación entre las variables epidemiológicas no es simple. La correlación más frecuentemente citada es que la prevalencia es igual a la incidencia en relación a los tiempos de duración, siendo esto de una simplificación excesiva.

La forma más conveniente para desarrollar un modelo IPM de la enfermedad, es asumir la independencia de todas las otras causas de muerte o daño. Bajo este supuesto, y debido a la falta de información más precisa, se utilizaron los valores de prevalencia y mortalidad disponibles a partir de varios estudios realizados en Chile, modelando estos datos en DisMod con el objetivo de estimar la incidencia de las enfermedades odontológicas como caries y enfermedad periodontal en el subgrupo de embazadas, como una alternativa a la observación directa que sería mucho más costosa y poco factible.

Las diferencias entre los valores reales y los obtenidos a través de un modelo podrían basarse en un supuesto implícito del uso de datos de un estudio de corte transversal en donde no hay ninguna secuencia temporal entre la incidencia y la supervivencia. Esto se debe a que en la estimación de la incidencia se basa en la prevalencia, que refleja las condiciones imperantes en el pasado (carga de la enfermedad).

A modo de conclusión, estos métodos son lo suficientemente generales como para ser utilizados en otras enfermedades del área de la salud, en donde la evaluación de la incidencia constituye un problema y su estudio en investigación de campo es muy costoso, de larga duración y por sobre todo éticamente inaceptable, aportando datos epidemiológicos como prevalencia, incidencia, duración de enfermedad y mortalidad específica. Estos datos son de gran utilidad en la planificación de los recursos sanitarios, en la toma de decisiones sobre nuevas intervenciones y en la evaluación de la eficiencia de estas (estudios de costo efectividad).

\section{CONFLICTOS DE INTERÉS}




\section{REFERENCIAS BIBLIOGRÁFICAS}

1. Barak S, Oettinger-Barak O, Oettinger M, Machtei EE, Peled M, Ohel G. Common oral manifestations during pregnancy: A review. Obstet Gynecol Surv, 2003 Sep; 58(9): 624-628.

2. Machuca G, Khoshfeiz O, Lacalle JR, Machuca C, Bullon P. The influence of general health and socio-cultural variables on the periodontal condition of pregnant women. J Periodontol, 1999 Jul; 70(7): 779-785.

3. Taani DQ, Habashneh R, Hammad MM, Batieha A. The periodontal status of pregnant women and its relationship with socio-demographic and clinical variables. J Oral Rehabil, 2003 Apr; 30(4): 440-445.

4. Tilakaratne A, Soory M, Ranasinghe AW, Corea SM, Ekanayake SL, de SM. Periodontal disease status during pregnancy and 3 months post-partum, in a rural population of Sri-Lankan women. J Clin Periodontol, 2000 Oct; 27(10): 787-792.

5. Laine MA. Effect of pregnancy on periodontal and dental health. Acta Odontol Scand, 2002 Oct; 60(5): 257-264.

6. Moss KL, Beck JD, Offenbacher S. Clinical risk factors associated with incidence and progression of periodontal conditions in pregnant women. $J$ Clin Periodontol, 2005 May; 32(5): 492-498.

7. Chaikin BS. Incidence of gingivitis in pregnancy. Quintessence Int, 1977; 8: 81-89. 8. Cuco G, Fernandez-Ballart J, Sala J, Viladrich C, Iranzo R, Vila J, Arija V. Dietary patterns and associated lifestyles in preconception, pregnancy and postpartum. Eur J Clin Nutr, 2006 Mar; 60(3): 364-371.

9. Rakchanok N, Amporn D, Yoshida Y, Harun-Or-Rashid M, Sakamoto J. Dental caries and gingivitis among pregnant and non-pregnant women in Chiang Mai, Thailand. Nagoya J Med Sci, 2010 Feb; 72(1-2): 43-50.
10. Papp E, Kengyeli I, Banoczy J, Csordas T. Longitudinal study of the dental status of pregnant women under prenatal care. Fogorv Sz, 1990 Jul; 83(7): 199-204. 11. Ruiz G, Gómez M, Rodríguez R. Relación entre la prevalencia de caries dental y embarazo. Revista ADM, 2002; 59(1): 5-9.

12. Kruijshaar ME, Barendregt JJ, Hoeymans N. The use of models in the estimation of disease epidemiology. Bull World Health Organ, 2002; 80(8): 622-628.

13. Barendregt JJ, Van Oortmarssen GJ, Vos T, Murray CJ. A generic model for the assessment of disease epidemiology: The computational basis of DisMod II. Popul Health Metr, 2003 Apr; 1(1): 4.

14. Instituto Nacional de Estadistica. http://www.ine.cl/. 2009. Ref Type: Electronic Citation.

15. Ministerio de Salud de Chile. Encuesta Nacional de Salud. http://epi.minsal.cl/ epi/html/invest/ENS/ENS.htm. 2003. Ref Type: Electronic Citation.

16. Ministerio de Salud de Chile. Estudio carga enfermedad y carga atribuible. http:// epi.minsal.cl/epi/html/invest/cargaenf2008/Informe\%20final\%20carga Enf 2007. pdf. 2007. Ref Type: Electronic Citation.

17. World Health Organization. International Classification of Disease (ICD). http:// www.who.int/classifications/icd/en/. 2010. Ref Type: Electronic Citation.

18. Barendregt JJ, Baan CA, Bonneux L. An indirect estimate of the incidence of non-insulin-dependent diabetes mellitus. Epidemiology, 2000 May; 11(3): 274-279. 